\title{
ATLAS: Status and First Results
}

\author{
Daniel R. Tovey ${ }^{a}$ \\ on behalf of the ATLAS Collaboration. \\ a Department of Physics and Astronomy, University of Sheffield, Hounsfield Road, Sheffield S3 7RH, UK \\ Recent results from the ATLAS experiment at the CERN Large Hadron Collider are presented. The experiment \\ has been functioning with high efficiency since the start of LHC data-taking, at proton-proton centre-of-mass \\ energies of $900 \mathrm{GeV}$ and $2.36 \mathrm{TeV}$ (2009) and $7 \mathrm{TeV}$ (2010). This paper provides a brief overview of the highlights \\ of recent detector performance and physics measurements.
}

\section{INTRODUCTION}

The ATLAS detector [1] is a large general purpose detector operating at the CERN Large Hadron Collider (LHC) [2]. The first ATLAS physics results dealing with charged particle multiplicities in $900 \mathrm{GeV}$ proton-proton collision were obtained with data from the initial 2009 LHC run [3]. Following a brief run at $2.36 \mathrm{TeV}$ and a planned shut-down, LHC data-taking recommenced in 2010 at a $p p$ centre-of-mass energy of $7 \mathrm{TeV}$. Rapid progress in 're-discovering' the Standard Model is being made with this data-set, enabled by excellent detector performance and stability. Among the highlights of this physics programme have been the observation of high $p_{T}$ jet events, reconstruction of $J / \psi \rightarrow \mu^{+} \mu^{-}$decays, and measurement of the cross sections for production of $W$ and $Z$ bosons at $7 \mathrm{TeV}$.

\section{THE ATLAS DETECTOR}

The ATLAS detector covers almost the whole solid angle around the nominal LHC collision point with layers of tracking detectors (the "inner detector'), calorimeters and muon chambers.

The ATLAS inner detector has full coverage in $\phi$ and covers the pseudorapidity range $|\eta|<$ 2.5. It consists of a silicon pixel detector (Pixel), a silicon microstrip detector ( $\mathrm{SCT}$ ) and a transition radiation tracker (TRT). These detectors cover a sensitive radial distance from the interaction point of $50.5-150 \mathrm{~mm}, 299-560 \mathrm{~mm}$ and
563-1066 $\mathrm{mm}$, respectively, and are immersed in a $2 \mathrm{~T}$ axial magnetic field. The inner detector barrel (end-cap) parts consist of $3(2 \times 3)$ pixel layers, $4(2 \times 9)$ double-layers of single-sided silicon microstrips with a 40 mrad stereo angle, and $73(2 \times 160)$ layers of TRT straws. These detectors have position resolutions of typically 10,17 and $130 \mu \mathrm{m}$ for the $R-\phi$ co-ordinate and, in the case of the Pixel and SCT, 115 and $580 \mu \mathrm{m}$ for the second measured co-ordinate. A track from a particle traversing the barrel detector would typically have 11 silicon hits (3 pixel clusters and 8 strip clusters), and more than 30 straw hits.

High granularity liquid-argon (LAr) electromagnetic sampling calorimeters, with excellent performance in terms of energy and position resolution, cover the pseudorapidity range of up to $|\eta|$ $=4.9$. The hadronic calorimetry in the range $|\eta|$ $<1.7$ is provided by a scintillator-tile calorimeter, which is separated into a large barrel and two smaller extended barrel cylinders, one on either side of the central barrel.

The calorimeter is surrounded by the muon spectrometer. The air-core toroid system, with a long barrel and two inserted end-cap magnets, generates strong bending power in a large volume within a light and open structure. Multiplescattering effects are thereby minimised, and excellent muon momentum resolution is achieved with three layers of high precision tracking chambers. The muon instrumentation includes, as a key component, trigger chambers with timing resolution of the order of 1.5-4 ns. The muon spec- 
trometer defines the overall dimensions of the ATLAS detector.

\section{DATA-TAKING AND TRIGGER}

As of $25^{\text {th }}$ July 2010 ATLAS had recorded approximately $340 \mathrm{nb}^{-1}$ of data under LHC stable beam conditions (which means that it is safe for ATLAS to turn on the full inner detector). The maximum peak luminosity as measured in ATLAS was $L=1.6 \times 10^{30} \mathrm{~cm}^{-2} s^{-1}$. The operational fraction of all ATLAS detector and trigger systems was close to $100 \%$ at all times.

The ATLAS detector has a three-level trigger system: Level 1 (L1), Level 2 (L2) and Event Filter (EF). The L2 and EF systems are collectively referred to as the High Level Trigger (HLT). The main physics trigger during the 2009 data taking relied on the L1 signals from the Beam Pickup Timing devices (BPTX) and the Minimum Bias Trigger Scintillators (MBTS). The BPTX are composed of beam pick-ups attached to the beam pipe at $z=175 \mathrm{~m}$ from the centre of the ATLAS detector. The MBTS are mounted at each end of the detector in front of the liquidargon end-cap calorimeter cryostat at $z=3.56 \mathrm{~m}$. Most data has been acquired during the 2010 run at $\sqrt{s}=7 \mathrm{TeV}$, using trigger menus appropriate to the instantaneous luminosity conditions. Initially only L1 triggers have been used with typical thresholds of order $3 \mathrm{GeV}$ (EM objects), 5-15 $\mathrm{GeV}$ (jets) and $0 \mathrm{GeV}$ (muons). As the trigger rates have increased thresholds have been raised or, as in the case of EM triggers, HLT rejection has been applied.

\section{DETECTOR AND COMBINED PER- FORMANCE HIGHLIGHTS}

ATLAS has already produced an impressive number of detector and combined performance results using the 2009 and 2010 collision data. This section can only show a small selection of these results.

The Pixel detector has an analogue read-out and can therefore measure the energy loss of traversing particles. Fig. 1(top) shows the energy loss versus the charge times the momentum of the
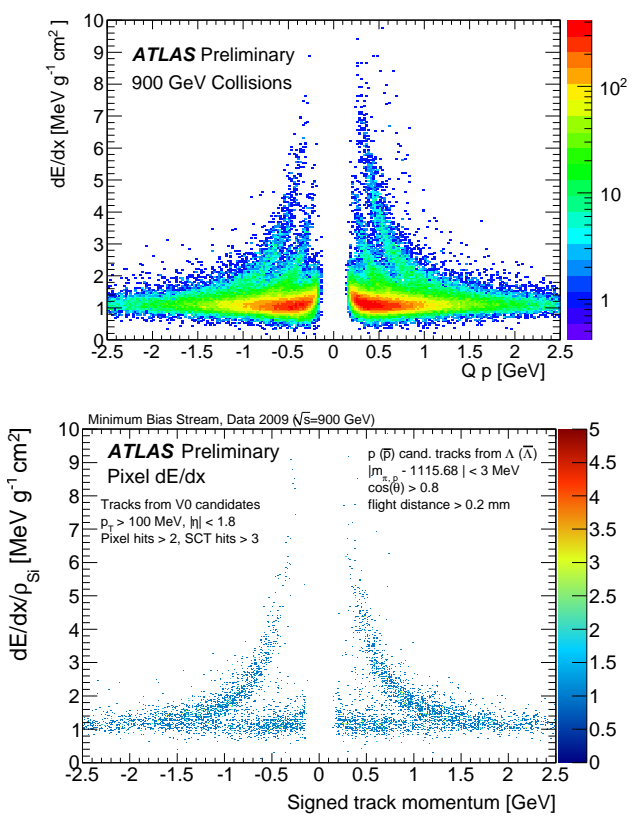

Figure 1. Top: energy loss in the pixel detector for all reconstructed tracks. The bands for pions, kaons and protons are clearly visible. Bottom: a pre-selection of tracks using only the proton of $\Lambda \rightarrow p \pi^{-}\left(\right.$and $\left.\bar{\Lambda} \rightarrow \bar{p} \pi^{+}\right)$removes the kaon band.

traversing particles. The bands for protons, kaons and pions can clearly be seen. A cross-check has been made by reconstructing the decay of particles such as $\Lambda \rightarrow p \pi^{-}$as shown in Fig. 1(bottom). When only plotting the energy loss for the decay products of the $\Lambda$ (and $\bar{\Lambda}$ ) only the bands for protons and pions remain.

The TRT has the ability to separate electrons and pions by measuring the photon radiation of the traversing particle. The intensity of this transition radiation is proportional to the Lorentz factor $\gamma=E / m_{0} c^{2}$ of the traversing particle. Fig. 2(top) shows the probability for producing so-called high threshold hits which are due to transition radiation in the TRT, for data and Monte Carlo. Pions in the momentum range 1 - $10 \mathrm{GeV}$ radiate far fewer photons than electrons in the same momentum range due to the 

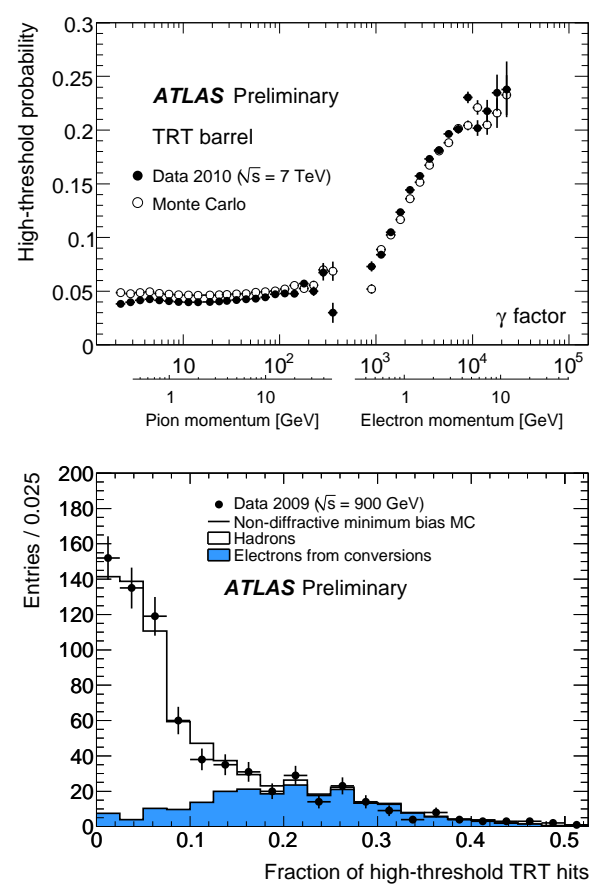

Figure 2. Top: the probability of a high-threshold hit in the TRT barrel as a function of the Lorentz factor $\gamma=E / m_{0} c^{2}$. Bottom: fraction of high threshold hits in the TRT for all reconstructed tracks in data and Monte Carlo.

large mass difference between the two particles. A cross-check of this can be made by using identified electrons from photon conversions $\left(\gamma \rightarrow e^{+} e^{-}\right)$. Fig. 2(bottom) shows the fraction of high threshold hits for all reconstructed tracks in data and Monte Carlo. The shaded area towards higher fractions of high threshold hits is entirely due to electrons from photon conversions.

The reconstruction of well known particle decays such as $K_{S}^{0} \rightarrow \pi^{+} \pi^{-}, \Lambda \rightarrow p \pi^{-}$or $\bar{\Lambda} \rightarrow \bar{p} \pi^{+}$ is a powerful tool for understanding and validating the performance of the detector [4]. Fig. 3 shows the reconstructed invariant mass spectra of the $K_{S}^{0}$ and the $\Lambda$. The mean values of the reconstructed particle masses for the $K_{S}^{0}, \Lambda$ and $\bar{\Lambda}$ (the latter is not shown) agree very well with
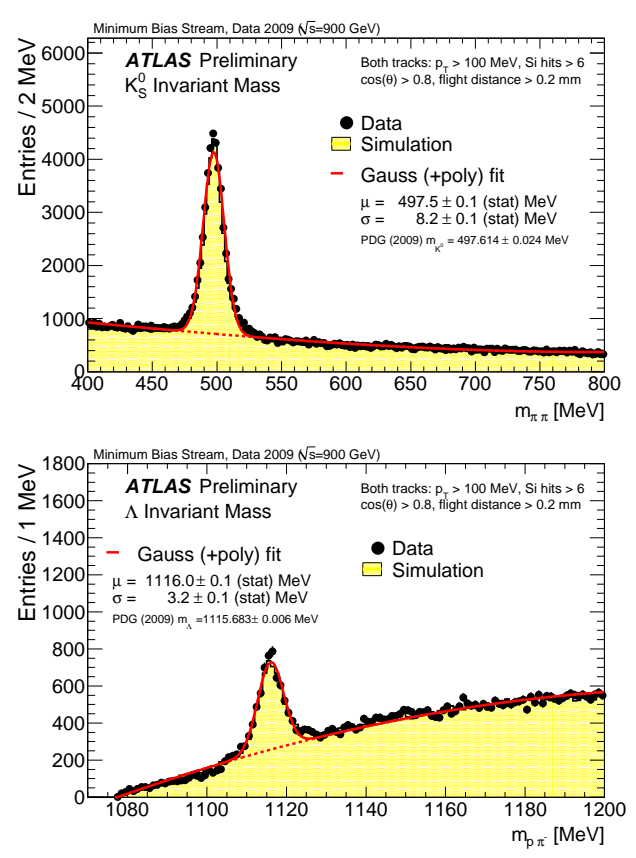

Figure 3. Reconstructed invariant mass spectra of $K_{S}^{0} \rightarrow \pi^{+} \pi^{-}$(top) and $\Lambda \rightarrow p \pi^{-}$(bottom).

the world averages [5]. The widths of the distributions show very good agreement between data and Monte Carlo. This underlines the good understanding of the detector (e.g. the material in the inner detector) at this early stage of data taking.

The electromagnetic calorimeter measures the energy of electrons and photons. Comparison between data and Monte Carlo estimates of its response to single isolated tracks permits assessment of systematic uncertainties in the understanding of calorimeter performance and the amount and distribution of material in the inner detector. Fig. 4 shows the energy deposited in the calorimeter over the momentum of a track as measured in the inner detector in the barrel region $|\eta|<0.6$ for isolated hadrons for data and Monte Carlo. The momentum of reconstructed tracks was required to lie in the range 1.2-1.8 $\mathrm{GeV}$. An isolation requirement of no other tracks 


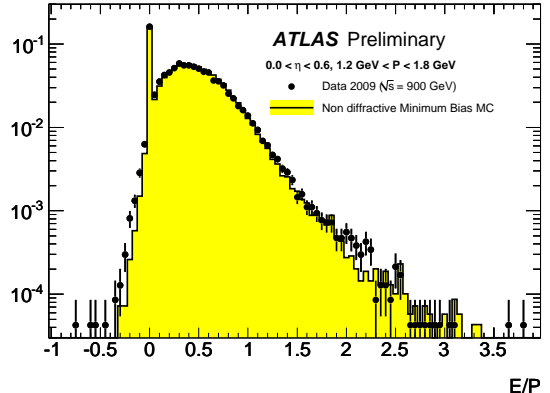

Figure 4 . The energy deposited in the calorimeter over the momentum, as measured in the inner detector, of an isolated track. Tracks possess $|\eta|=[0,0.6]$ and $p=[1.2,1.8] \mathrm{GeV}$.

within a cone in $\eta$ and $\phi$ of 0.4 was made. The peak at zero is due to particles that stopped in the inner detector (e.g. due to hadronic interactions) and hence did not deposit any energy in the calorimeter. The agreement between simulation and data is remarkable and also reflects a good understanding of the material in the inner detector in the simulation.

The muon spectrometer will play an important role in triggering on interesting physics channels at higher centre-of-mass energies. An early indicator of its excellent performance has been the precision with which the $J / \psi \rightarrow \mu^{+} \mu^{-}$decay process can be reconstructed, as discussed in the next section.

\section{PHYSICS HIGHLIGHTS}

\subsection{Observation of $J / \psi \rightarrow \mu^{+} \mu^{-}$}

The decay $J / \psi \rightarrow \mu^{+} \mu^{-}$has been observed in ATLAS data using combined information from the muon spectrometer and the inner detector [6]. A clear peak was observed using $6.4 \pm 1.3 \mathrm{nb}^{-1}$ of data (Fig. 5). The peak was fitted using an unbinned maximum likelihood method; this yielded an overall mean of $3.095 \pm 0.004 \mathrm{GeV}$, which is in agreement with the PDG value for the $J / \psi$ mass [5] within statistical uncertainty. This close match is a consequence of the work done with

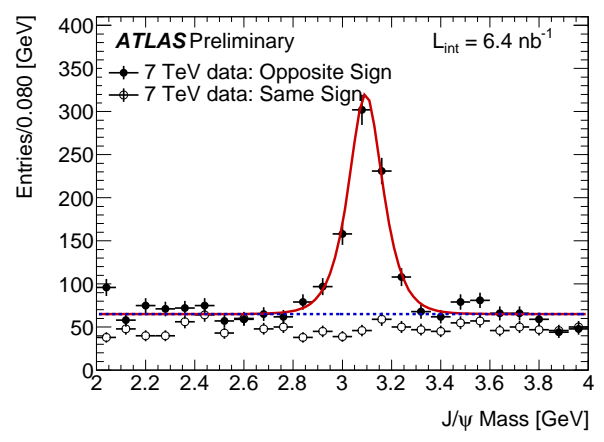

Figure 5. Invariant mass distribution of reconstructed $J / \psi \rightarrow \mu^{+} \mu^{-}$candidates after vertexing cuts. Same sign combinations (open circles) are superimposed.

cosmic ray events and earlier collisions to attain a good understanding of the ATLAS detector. The signal resolution was $82 \pm 7 \mathrm{MeV}$, in line with Monte Carlo expectations. The mass resolution varies with the pseudorapidity of the muons and this variation is in agreement with Monte Carlo within statistical uncertainty. The process of finding the $J / \psi \rightarrow \mu^{+} \mu^{-}$decay vertex, which will be required for all subsequent physics studies on $J / \psi$, was not found to affect the measured mass or its resolution beyond statistical uncertainties. After all cuts the number of observed $J / \psi \rightarrow \mu^{+} \mu^{-}$decays was $612 \pm 34$, over a background of $332 \pm 9$ candidates.

\subsection{Measurement of jet cross sections}

The inclusive jet and dijet cross sections have been measured using $17 \mathrm{nb}^{-1}$ of $7 \mathrm{TeV}$ collisions [7]. The measured cross sections extend up to jet $p_{T}$ of $500 \mathrm{GeV}$ and dijet masses of $2 \mathrm{TeV}$. Leading logarithmic parton-shower Monte Carlo programs provide a reasonable description of the energy flow around the jets, and of the shapes of the cross sections. The measurements are well described by fixed-order NLO perturbative QCD calculations corrected for non-perturbative effects (Fig. 6). 


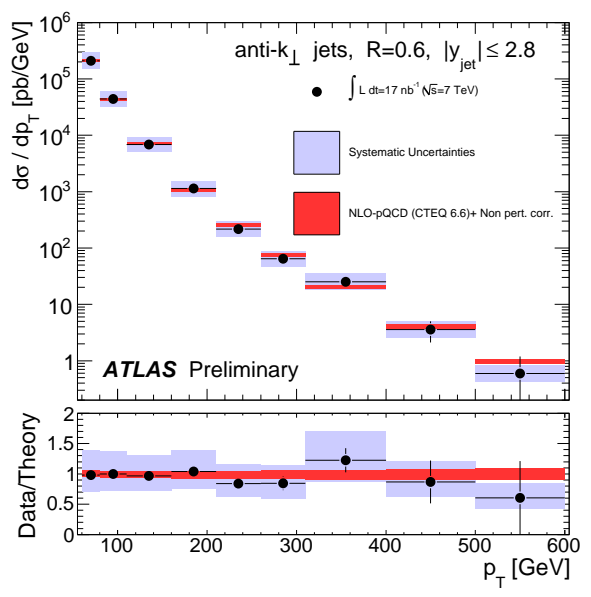

Figure 6. Inclusive jet differential cross section as a function of jet $p_{T}$ integrated over the region $|y|<2.8$ for jets identified using the anti- $k_{T}$ algorithm with $R=0.6$.

\subsection{Measurement of the cross sections for production of $W$ and $Z$ bosons}

The cross section for $W$ boson production in $p p$ collisions at $\sqrt{s}=7 \mathrm{TeV}$ has been measured using leptonic decays to $e \nu$ (Fig. 7 (top)) and $\mu \nu$ (Fig. 7(bottom)) [8]. $118 \mathrm{~W}$ boson candidates were observed in data corresponding to a total integrated luminosity of $16.9 \mathrm{nb}^{-1}$ for the $W \rightarrow e \nu$ channel, and $16.6 \mathrm{nb}^{-1}$ for the $W \rightarrow \mu \nu$ channel. The total inclusive $W$ boson production cross section times the leptonic branching ratio was measured to be $(8.5 \pm 1.3$ (stat) \pm 0.7 (syst) \pm 0.9 (lumi) ) nb for the $W \rightarrow e \nu$ channel and (10.3 \pm 1.3 (stat) \pm 0.8 (syst) \pm 1.1 (lumi) ) nb for the $W \rightarrow$ $\mu \nu$ channel. This constitutes the first $W$ boson cross section measurement by ATLAS and the result obtained is in agreement with theoretical calculations based on NNLO QCD (Fig. 8(top)). In addition the expected charge asymmetry between the cross section for $W^{+}$and $W^{-}$boson production is experimentally confirmed.

The cross section for $Z$ boson production in $p p$ collisions at $\sqrt{s}=7 \mathrm{TeV}$ has also been measured using a somewhat larger data sample correspond- ing to approximately $225 \mathrm{nb}^{-1}$ [9]. In this sample a total of 125 candidate $Z \rightarrow e^{+} e^{-}$or $Z \rightarrow \mu^{+} \mu^{-}$ events were observed after all cuts. The total inclusive $Z$ boson production cross section times the charged leptonic branching ratio was measured to be $[0.72 \pm 0.11$ (stat) \pm 0.10 (syst) \pm 0.08 (lumi)] $\mathrm{nb}$ for the $Z \rightarrow e^{+} e^{-}$channel and $[0.89 \pm 0.10$ (stat) \pm 0.07 (syst) \pm 0.10 (lumi)] $\mathrm{nb}$ for the $Z \rightarrow \mu^{+} \mu^{-}$channel, resulting in a combined result of $[0.83 \pm 0.07$ (stat) \pm 0.06 (syst) \pm 0.09 (lumi) $] \mathrm{nb}$ all measured within the invariant mass window $66<m_{\ell \ell}<116 \mathrm{GeV}$. This constitutes the first $Z$ boson cross section measurement by ATLAS and the result obtained is in agreement with theoretical calculations based on NNLO QCD (Fig. 8(bottom)).

\section{CONCLUSIONS}

First performance and physics results from the ATLAS detector at the LHC have been presented. These results show that the ATLAS detector is performing extremely well, benefiting from many years of preparation with test beams, cosmic ray runs, and Monte Carlo studies. The results bode well for future measurements and discoveries.

\section{REFERENCES}

1. The ATLAS Collaboration, JINST 3 (2008) S08003.

2. L. Evans and P. Bryant, JINST 3 (2008) S08001.

3. The ATLAS Collaboration, Phys. Lett. B 688 (2010) 21 [arXiv:1003.3124].

4. The ATLAS Collaboration, ATLAS-CONF2010-033

5. C. Amsler et al. [Particle Data Group], Phys. Lett. B 667 (2008) 1.

6. The ATLAS Collaboration, ATLAS-CONF2010-045

7. The ATLAS Collaboration, ATLAS-CONF2010-050

8. The ATLAS Collaboration, ATLAS-CONF2010-051

9. The ATLAS Collaboration, ATLAS-CONF2010-076 

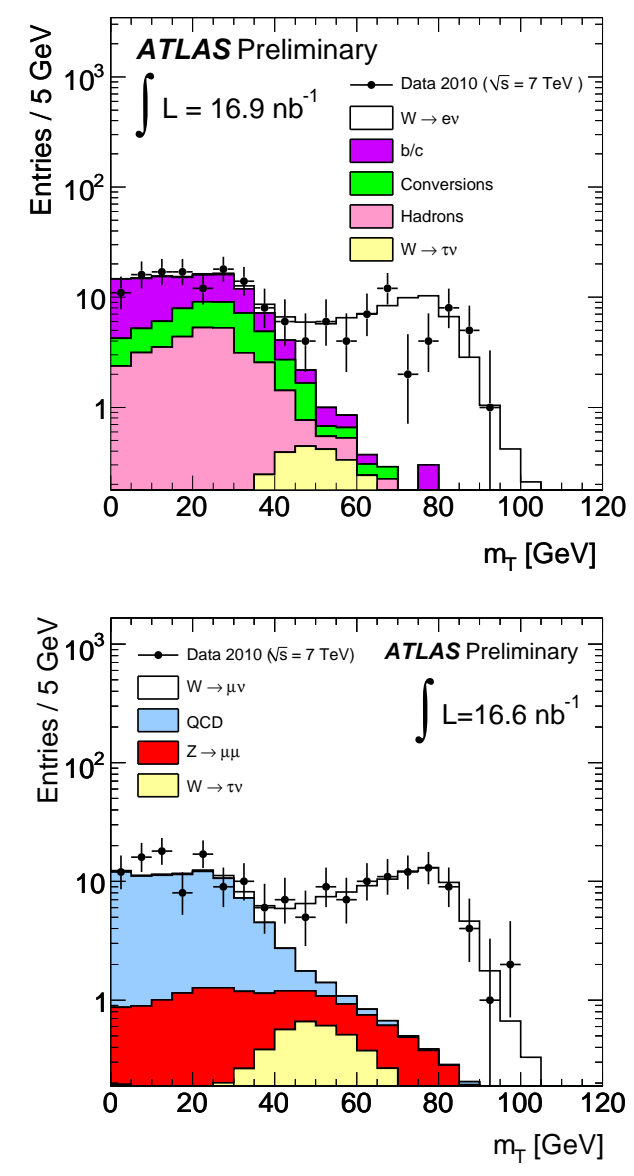

Figure 7. Transverse mass of the lepton- $E_{T}^{\text {miss }}$ system of selected $W$ boson candidate events without any cut on $E_{T}^{\text {miss }}$, for electron events (top) and muon events (bottom).
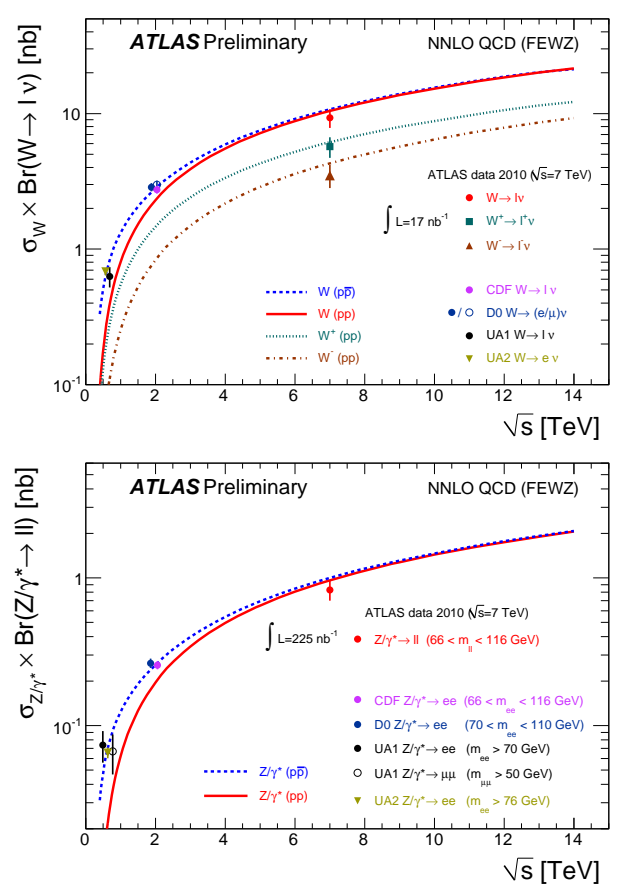

Figure 8. Top: the measured values of $\sigma_{W} \times B R$ $(W \rightarrow \ell \nu)$ for $W^{+}, W^{-}$and for their sum compared to the theoretical predictions based on NNLO QCD calculations. Bottom: the measured values of $\sigma_{Z} \times B R\left(Z \rightarrow \ell^{+} \ell^{-}\right)$where the electron and muon channels have been combined, compared to the theoretical predictions based on NNLO QCD calculations. 